\title{
Effect of eccentricity on natural convection in fluid-saturated porous media in an elliptical annulus
}

\author{
Hichem Boulechfar, Mahfoud Djezzar a And Amel Labed \\ Energetic Physics Laboratory, Faculty of Exact Sciences, Constantine University 1, Algeria
}

Received 14 April 2014, Accepted 6 December 2014

\begin{abstract}
In this paper, we summarize a numerical study of two-dimensional natural convection in an annular elliptical space fluid-saturated porous, by solving numerically the mass balance equations, momentum and energy, using Darcy's law, Boussinesq approximation, vorticity-stream function formulation and the finite volumes method for the discretization of partial derivative equations. Both walls delimiting the annular space are maintained at two uniform different temperatures. The external parameters considered are the eccentricity of the inner elliptic wall $(0.55,0.688,0.86,0.9$ and 0.999$)$ and Rayleigh-Darcy number $\left(R a_{m}=500\right)$. The results indicate that there are two main modes of natural convection: natural convection with only two cells and with more cells, their description is given in detail. The average equivalent conductivity is presented in terms of external parameters and allows us to see that it increases when the internal wall eccentricity increases.
\end{abstract}

Key words: Natural convection / porous media / elliptical annulus / vorticity stream function formulation / Newtonian fluid

\section{Introduction}

During recent years a considerable research effort has been devoted to the study of heat transfer induced by natural convection in a porous medium saturated by a fluid. Interest in these phenomena of natural convection is due to many potential applications in engineering. These applications include the extraction of geothermal energy, the dispersion of pollutants in aquifers, security issues in the heart of nuclear reactors, thermal insulation of buildings, etc.

Philip [1] developed exact solutions for small Rayleigh number free convection in 2-D porous cavities of various shapes due to a uniform temperature gradient normal to the gravitational field. In this study the theorem is established that such flows are independent of cavity orientation. The exact solutions in detail for rectangular and elliptical cavities with $\lambda$ (ratio of long to short axis) arbitrary, and for equilateral triangular cavities, are presented. The critical Rayleigh number for applicability of the analysis is about 1 for cavities with long axis horizontal, and about $\lambda^{1 / 2}$ with long axis vertical. Elshamy and Ozisik [2] studied numerically a steady-state natural convection for air bounded by two confocal horizontal elliptical cylinders for the case of inner hot and outer

${ }^{a}$ Corresponding author: mdjezzar@umc.edu.dz cold isothermal surfaces. The local and average Nusselt numbers were determined for different values of Rayleigh number for different eccentricities of the inner surface.

Charrier-Mojtabi [3] carried a numerical investigation of two-dimensional and three-dimensional free convection flows in a saturated porous horizontal annulus heated from the inner surface, using a Fourier-Galerkin approximation for the periodic azimuthal and axial directions and a collocation-Chebyshev approximation in the confined radial direction. The numerical algorithm integrates the Darcy-Boussinesq's equations formulated in terms of pressure and temperature. This method gives an accurate description of the 2-D multicellular structures for a large range of Rayleigh number and radii ratio. Bifurcation points between 2-D unicellular flows and either 2-D multicellular or 3-D flows are also determined numerically. Mota et al. [4] solved the two-dimensional DarcyBoussinesq equations, governing natural convection heat transfer in a saturated porous medium, in generalized orthogonal coordinates, using high-order compact finite differences on a very fine grid. The mesh is generated numerically using the orthogonal trajectory method. The code is applied to horizontal eccentric elliptic annuli containing saturated porous media. The judicious stretching of one of the annular walls in the horizontal direction reduces the heat losses with respect to a concentric cylindrical annulus with the same amount of insulating material. 


\section{Nomenclature}

\begin{tabular}{|c|c|c|}
\hline & & \\
\hline$a$ & Thermal diffusivity, & $\mathrm{m}^{2} \cdot \mathrm{s}^{-1}$ \\
\hline$A$ & Elliptic cylinder major axis, & $\mathrm{m}$ \\
\hline$B$ & Elliptic cylinder minor axis, & $\mathrm{m}$ \\
\hline$c$ & Constant defined in the elliptic coordinates, & \\
\hline $\begin{array}{l}c_{p} \\
D a\end{array}$ & $\begin{array}{l}\text { Specific heat at constant pressure, } \\
\text { Darcy number }\end{array}$ & $\mathrm{J} \cdot \mathrm{kg}^{-1} \cdot \mathrm{K}^{-1}$ \\
\hline$e$ & Elliptic cylinder eccentricity, & {$\left[=\sqrt{\frac{\left(A^{2}-B^{2}\right)}{A^{2}}}\right.$} \\
\hline$g$ & Gravitational acceleration, & $\mathrm{m} \cdot \mathrm{s}^{-2}$ \\
\hline$G r$ & Grashof number, $\left[=\mathrm{g} \beta c^{3}\left(T_{1}-T_{2}\right) / v^{-2}\right]$ & \\
\hline$h$ & Dimensional metric coefficient, & $\mathrm{m}$ \\
\hline$H$ & Dimensionless metric coefficient & \\
\hline$K$ & Porous medium permeability, & $\mathrm{m}^{2}$ \\
\hline$N u$ & Local Nusselt number & \\
\hline$P$ & Pressure, & N.m ${ }^{-2}$ \\
\hline $\operatorname{Pr}$ & Prandtl number, $\left[=\left(v \rho c_{p}\right) / \lambda\right]$ & \\
\hline$R a$ & Rayleigh number, $[=G r P r]$ & \\
\hline$R a_{m}$ & Rayleigh-Darcy number, $[=R a D a]$ & \\
\hline$t$ & Time, & $\mathrm{s}$ \\
\hline$T$ & Fluid's temperature, & $\mathrm{K}$ \\
\hline$T_{1}$ & Inner wall's temperature, & $\mathrm{K}$ \\
\hline$T_{2}$ & Outer wall's temperature, & $\mathrm{K}$ \\
\hline$u$ & Velocity component-coordinate $x$ & $\mathrm{~m} \cdot \mathrm{s}^{-1}$ \\
\hline$v$ & Velocity component-coordinate $y$ & $\mathrm{~m} \cdot \mathrm{s}^{-1}$ \\
\hline$U_{\eta}$ & Velocity component-coordinate $\eta$ & $\mathrm{m} \cdot \mathrm{s}^{-1}$ \\
\hline$V_{\theta}$ & Velocity component-coordinate $\theta$ & $\mathrm{m} \cdot \mathrm{s}^{-1}$ \\
\hline$\vec{V}$ & Velocity vector & $\mathrm{m} \cdot \mathrm{s}^{-1}$ \\
\hline$x, y$ & Cartesian coordinates & $\mathrm{m}$ \\
\hline & Greek letters & \\
\hline$\alpha$ & Inclination angle, & 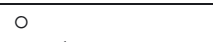 \\
\hline$\beta$ & Thermal expansion coefficient, & $\mathrm{K}^{-1}$ \\
\hline$\mu$ & Dynamic viscosity, & $\mathrm{kg} \cdot \mathrm{m}^{-1} \cdot \mathrm{s}^{-1}$ \\
\hline$\lambda$ & Thermal conductivity, & $\mathrm{W} \cdot \mathrm{m}^{-1} \cdot \mathrm{K}^{-1}$ \\
\hline$v$ & Kinematic viscosity, & $\mathrm{m}^{2} \cdot \mathrm{s}^{-1}$ \\
\hline$\sigma_{T}$ & Thermal capacity factor & \\
\hline$\Delta T$ & Temperature difference, $\left[=T_{1}-T_{2}\right]$ & $\mathrm{K}$ \\
\hline$\rho$ & Density, & $\mathrm{kg} \cdot \mathrm{m}^{-3}$ \\
\hline$\psi$ & Stream function, & $\mathrm{m}^{2} \cdot \mathrm{s}^{-1}$ \\
\hline$\eta, \theta$ & Elliptic coordinates & \\
\hline & Subscripts & \\
\hline 1 & Inner & \\
\hline 2 & Outer & \\
\hline$p$ & Porous & \\
\hline & Superscripts & \\
\hline+ & Dimensionless parameters & \\
\hline
\end{tabular}

Previous studies show that, under certain conditions, eccentric cylinders may lead to a more effective insulation than concentric ones. The results presented here provide an alternative approach to optimizing the heat transfer rate by a proper choice of the annular shape. Djezzar et al. $[5,6]$ expressed the Boussinesq equations of the laminar thermal and natural convection, in the case of permanent and bidimensional flow, in an annular space between two confocal elliptic cylinders. A new calculation code uses the finite volumes with the primitive functions (velocity-pressure formulation) using the elliptic coordinates system. Both effects of Rayleigh number and the geometry of the interior elliptic cylinder were examined. Leong and Lai [7] presented for natural convection in concentric cylinders with a porous sleeve, analytical solutions obtained through perturbation method and Fourier transform. The porous sleeve is press-fitted to the inner surface of the outer cylinder. Both the inner and outer cylinders are kept at constant temperatures with the inner surface at a slightly higher temperature than that of the outer. The main objective of this study is to investigate the buoyancy-induced flow as affected by the presence of the porous layer. They performed parametric study to investigate the effects of Rayleigh number, Darcy number, 
porous sleeve thickness, and relative thermal conductivity on the heat transfer results. Khanafer et al. [8] investigated a numerical natural convection heat transfer within a two-dimensional, horizontal annulus that is partially filled with a fluid-saturated porous medium. In addition, the porous sleeve is considered to be press fitted to the inner surface of the outer cylinder. Both cylinders are maintained at constant and uniform temperatures with the inner cylinder being subjected to a relatively higher temperature than the outer one. Moreover, the Forchheimer and Brinkman effects are taken into consideration when simulating the fluid motion inside the porous sleeve. Furthermore, the local thermal equilibrium condition is assumed to be applicable for this investigation. The working fluid is air while copper is used to represent the solid phase. The porosity is considered to be uniform and constant with $\varepsilon=0.9$. The effect of the porous sleeve on the buoyancy induced flow motion under steady-state condition was examined. Kumari and Nath [9] studied the unsteady natural convection flow from a horizontal cylindrical annulus filled with a non-Darcy porous medium .The unsteadiness in the problem arises due to the impulsive change in the wall temperature of the outer cylinder. The Navier-Stokes equations along with the energy equation governing the unsteady natural convection flow have been solved by the finite-volume method. The results show that the annulus completely filled with a porous medium has the best insulating effectiveness. The effect of porous medium Darcy number on the heat transfer is more pronounced than that of the Grashof number. Sankar et al. [10] investigated natural convection flows in a vertical annulus filled with a fluid-saturated porous medium, when the inner wall is subject to discrete heating. The outer wall is maintained isothermally at a lower temperature, while the top and bottom walls, and the unheated portions of the inner wall are kept adiabatic. Through the Brinkman-extended Darcy equation, the relative importance of discrete heating on natural convection in the porous annulus is examined. An implicit finite difference method has been used to solve the governing equations of the flow system. The analysis is carried out for a wide range of Rayleigh and Darcy numbers for different heat source lengths and locations. The numerical results reveal that an increase in the radius ratio, Rayleigh number and Darcy number increases the heat transfer, while the heat transfer decreases with an increase in the length of the heater.

Our contribution is to solve the problem of natural convection in porous media saturated with a fluid inside an annular elliptical geometry using the vorticity-stream function formulation in elliptical coordinates. The interest in considering an annular elliptical geometry is based on their adaptability to become either circular when the axis ratio approaches unity, or flat plate when the axis ratio approaches zero. This type of geometry can be found in a wide range of applications such the heat exchangers consisting of coaxial tubes. The inner tube wall can be heated by flowing a hot fluid in the inner space at a constant temperature in order to obtain a temperature

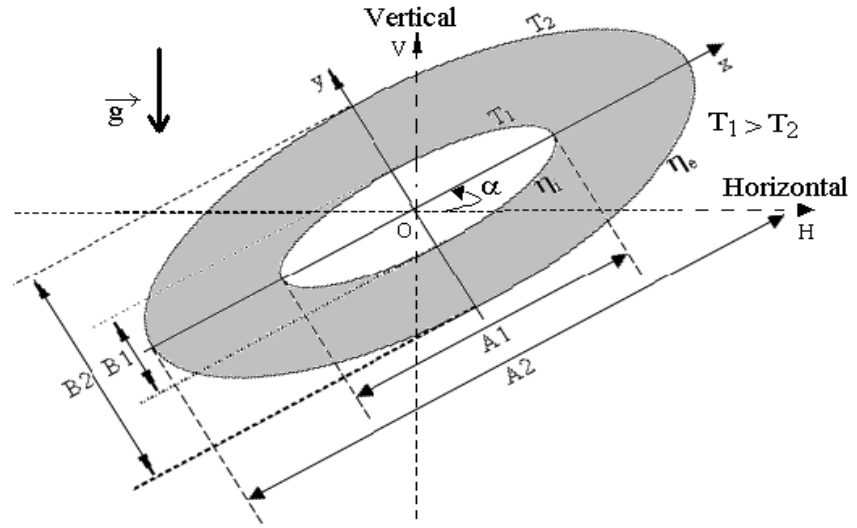

Fig. 1. Cross-section of the system.

gradient. Our work is dealing with the natural convection that occurs only in the annular space considered as a porous medium saturated with a fluid and bounded by two elliptical walls.

\section{Problem formulation and basic equation}

An annular elliptical space fluid-saturated porous and inclined is considered. Figure 1 represents a cross section of the system. Both elliptic internal and external walls are isothermal, kept at temperatures $T_{1}$ and $T_{2}$ respectively, with $T_{1}>T_{2}$.

The physical properties of the fluid are constant, except the density $\rho$ whose variations are at the origin of the natural convection. Viscous dissipation is neglected, just as the radiation (emissive properties of the two walls being neglected). We assume that the problem is bidimensionnal, steady and laminar.

In this space described above a natural convection occurs and it's subject to a numerical study. The natural convection equations within the framework of the Boussinesq approximation are written:

- Continuity equation

$$
\operatorname{div} \vec{V}=0
$$

- Momentum equation

The classic formulation of Darcy is used for writing the equation of motion:

$$
\vec{V}=-\frac{K}{\mu}(\nabla P-\rho \vec{g})
$$

- Heat equation

$$
\sigma_{T} \frac{\partial T}{\partial t}+(\vec{V} \cdot \overrightarrow{\operatorname{grad}}) T=\frac{\lambda_{p}}{\rho C_{p}} \nabla^{2} T
$$

It is convenient to define a reference frame such as the limits of the system result in constant values of the coordinates. The passage of the Cartesian coordinates $(x, y)$ to 
the elliptic coordinates $(\eta, \theta)$ is obtained by the following relations:

$$
\left.\begin{array}{l}
x=c \operatorname{ch}(\eta) \cdot \cos (\theta) \\
y=c \operatorname{sh}(\eta) \cdot \sin (\theta)
\end{array}\right\}
$$

(1)-(3) are written respectively in the elliptic coordinates using the metric coefficient $h$ :

$$
\begin{aligned}
h=h_{1}=h_{2}= & c\left(\operatorname{sh}^{2}(\eta)+\sin ^{2}(\theta)\right)^{1 / 2} \\
& \text { and } h_{3}=1 \quad \text { with } \quad c=\frac{A}{\operatorname{ch}(\eta)}=\frac{B}{\operatorname{sh}(\eta)}
\end{aligned}
$$

The continuity equation represented by (1) is re-written in the elliptic coordinates as following:

$$
\frac{\partial}{\partial \eta}\left(h V_{\eta}\right)+\frac{\partial}{\partial \theta}\left(h V_{\theta}\right)=0
$$

Projections of (2) after some long manipulations and by introducing the stream function $\psi$ give:

$$
\begin{gathered}
V_{\eta}=\frac{1}{h} \frac{\partial \psi}{\partial \theta}, \quad V_{\theta}=-\frac{1}{h} \frac{\partial \psi}{\partial \eta} \\
\frac{1}{h}\left[\frac{\partial^{2} \psi}{\partial \eta^{2}}+\frac{\partial^{2} \psi}{\partial \theta^{2}}\right]=-\frac{K g \beta}{v}([\cos (\alpha) F(\eta, \theta)-\sin (\alpha) G(\eta, \theta)] \\
\left.\times \frac{\partial T}{\partial \eta}-[\sin (\alpha) F(\eta, \theta)+\cos (\alpha) G(\eta, \theta)] \frac{\partial T}{\partial \theta}\right) \\
V_{\eta} \frac{\partial T}{\partial \eta}+V_{\theta} \frac{\partial T}{\partial \theta}=a\left(\frac{1}{h^{2}}\right)\left[\frac{\partial^{2} T}{\partial \eta^{2}}+\frac{\partial^{2} T}{\partial \theta^{2}}\right]
\end{gathered}
$$

$V_{\eta}$ and $V_{\theta}$ are the velocity components in the directions $\eta$ and $\theta$.

The coefficients $F(\eta, \theta), G(\eta, \theta)$ used in (6) are given by:

$$
\begin{aligned}
& F(\eta, \theta)=\frac{\operatorname{sh}(\eta) \cos (\theta)}{\left(\operatorname{sh}^{2}(\eta)+\sin ^{2}(\theta)\right)^{1 / 2}} \\
& \text { and } G(\eta, \theta)=\frac{\operatorname{ch}(\eta) \sin (\theta)}{\left(\operatorname{sh}^{2}(\eta)+\sin ^{2}(\theta)\right)^{1 / 2}}
\end{aligned}
$$

The characteristic quantities used for the dimensionless problem are: $\Delta T=T_{1}-T_{2}$ between the inner and the outer elliptic cylinder is the characteristic temperature, the focal length $c$ in elliptic coordinates is the reference length and the thermal diffusivity of fluid $a$ is the characteristic velocity. The dimensionless mathematical model obtained is:

$$
\begin{gathered}
\frac{\partial}{\partial \eta}\left(H V_{\eta}^{+}\right)+\frac{\partial}{\partial \theta}\left(H V_{\theta}^{+}\right)=0 \\
H V_{\eta}^{+} \frac{\partial T^{+}}{\partial \eta}+H V_{\theta}^{+} \frac{\partial T^{+}}{\partial \theta}=\left[\frac{\partial^{2} T^{+}}{\partial \eta^{2}}+\frac{\partial^{2} T^{+}}{\partial \theta^{2}}\right]
\end{gathered}
$$

$$
\begin{array}{r}
{\left[\frac{\partial^{2} \psi^{+}}{\partial \eta^{2}}+\frac{\partial^{2} \psi^{+}}{\partial \theta^{2}}\right]=-R a_{m} H[[\cos (\alpha) F(\eta, \theta)} \\
-\sin (\alpha) G(\eta, \theta)] \frac{\partial T^{+}}{\partial \eta}-[\sin (\alpha) F(\eta, \theta) \\
\left.+\cos (\alpha) G(\eta, \theta)] \frac{\partial T^{+}}{\partial \theta}\right]
\end{array}
$$

where $V_{\eta}^{+}$and $V_{\theta}^{+}$the components of the dimensionless velocity are defined by:

$$
V_{\eta}^{+}=\frac{1}{H} \frac{\partial \psi^{+}}{\partial \theta} \quad \text { and } \quad V_{\theta}^{+}=-\frac{1}{H} \frac{\partial \psi^{+}}{\partial \eta}
$$

where $H=h / c$ is the dimensionless metric coefficient.

$R a_{m}$ the Rayleigh-Darcy number is defined by: $R a_{m}=$ RaDa.

The boundary conditions are the following ones:

Hot inner wall $\left(\eta=\eta_{i}=\right.$ cst. $)$ :

$$
V_{\eta}^{+}=V_{\theta}^{+}=\frac{\partial \psi^{+}}{\partial \eta}=\frac{\partial \psi^{+}}{\partial \theta}=0 \quad \text { and } \quad T_{1}^{+}=1
$$

Cold outer wall $\left(\eta=\eta_{\mathrm{e}}=\mathrm{cst}\right)$ :

$$
V_{\eta}^{+}=V_{\theta}^{+}=\frac{\partial \psi^{+}}{\partial \eta}=\frac{\partial \psi^{+}}{\partial \theta}=0 \quad \text { and } \quad T_{2}^{+}=0
$$

\section{Numerical method}

To solve (9) with the associated boundary conditions, we consider a numerical solution by the finite volumes method, exposed by [11]. The power law scheme was used for the discretization. For (10), we consider a numerical solution by the centered differences method. The iterative method used for the numerical solution of algebraic system of equations is the Gauss-Seidel with an underrelaxation process. Figure 2 shows the physical and computational domain, once the temperature distribution is available; the local Nusselt number in the physical domain is defined as:

$$
N u=-\left.\frac{1}{h} \frac{\partial T^{+}}{\partial \eta}\right|_{\eta=\mathrm{cst}}
$$

\section{Results and discussion}

For the validation of the computational problem, we compared our results with those of literature: the numerical simulation of two- and three-dimensional free convection flows in a horizontal porous annulus using a pressure and temperature formulation of [3] and the natural convection heat transfer in horizontal eccentric elliptic annuli containing saturated porous media of [4]. The grid dependence has been investigated using different mesh sizes before settling to a mesh size of $(61 \times 81)$. Our objective is to analyze the effect of eccentricity on heat transfer and flow. For this reason, we presented the isotherms and streamlines for different values of eccentricities for determined value of Rayleigh-Darcy number $R a_{m}=500$ and when the inclination $\alpha=0^{\circ}$. 


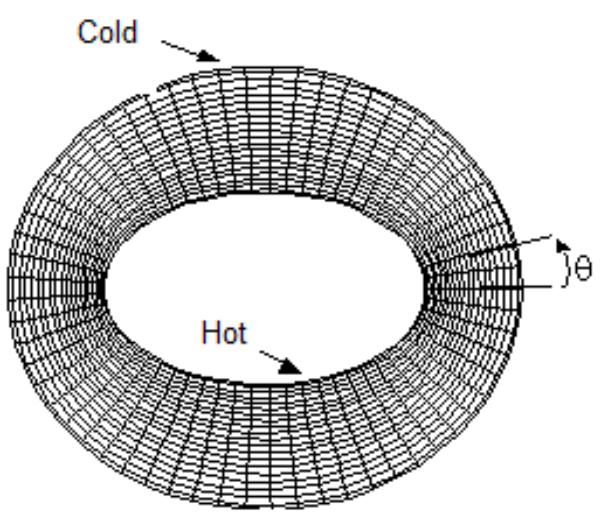

Physical Domain

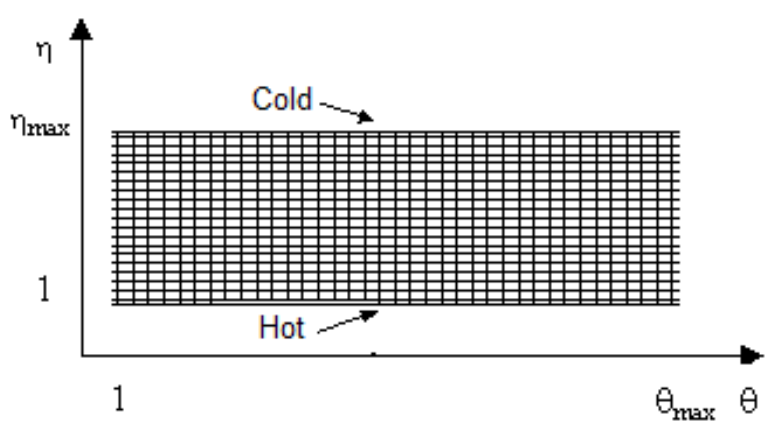

Computationnal Domain

Fig. 2. Physical and computational domain.

\subsection{Influence of the internal well eccentricity}

We consider five annular spaces characterised by the eccentricity of the internal elliptic cylinder $\left(e_{1}=0.55\right)$, $\left(e_{1}=0.688\right),\left(e_{1}=0.86\right),\left(e_{1}=0.9\right)$ and $\left(e_{1}=0.999\right)$.

The eccentricity of the external cylinder is maintained constant $\left(e_{2}=0.5\right)$. We use only one value of RayleighDarcy number $R a_{m}=500$ and the inclination chosen for the calculation is $\alpha=0^{\circ}$. The fluid is assumed as air, so the number of Prandtl is supposed be constant and equal to 0.702 .

Figure 3 for the value of Rayleigh-Darcy number considered shows that when $e_{1}=0.55$ the free space between two surfaces of exchange is very small, it favours the conductive transfer. With increasing the value of the eccentricity for the internal ellipse in Figure 4 the free space will be relatively expanded to facilitate the transfer by convection that is relatively low.

This transfer by convection becomes more important for larger annular spaces Figures 5-7, which shows that increasing the amount of internal eccentricity $e_{1}$, therefore causes an increase in the free space between the two surfaces exchange, which results in an intensification of the natural convection.

\subsection{Influence of the internal eccentricity on average equivalent conductivity}

Since this study examines the natural convection in an annulus whose geometry varies with eccentricity, the equivalent conductivity is the most suitable parameter with which to compare the surface heat transfers of the various annular spaces. The equivalent conductivity is defined as:

$$
\lambda_{e q}^{*}=\frac{\left.\frac{\partial T^{*}}{\partial \eta}\right|_{\text {Conduction+Convection }}}{\left.\frac{\partial T^{*}}{\partial \eta}\right|_{\text {Conduction }}}
$$

The Nusselt number is proportional to the overall value of heat transfer rate which consists of both modes conduction and convection; whereas the equivalent conductivity

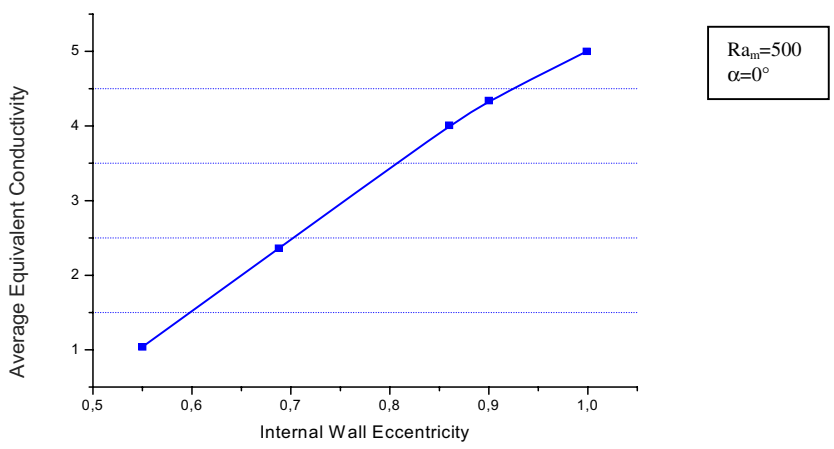

Fig. 8. Average equivalent conductivity on the internal wall.

represents the ratio of the total heat transfer between the inner and outer cylinders, the Nusselt number is not a good indicator of heat transfer when comparing different geometries.

The local equivalent conductivity is defined as the ratio of the local Nusselt number on the surface over which a fluid is moving to the local Nusselt number which would be calculated if the fluid was quiescent.

The overall equivalent conductivity is given by the ratio of the average Nusselt numbers for either case.

Figure 8 shows that the average equivalent conductivity values on the internal wall increase with the increasing of the internal eccentricity. For a narrow annular space, both walls are very close to each other which give a transfer by pseudo-conduction higher compared to the transfer by convection. By increasing the eccentricity of the inner elliptical tube which tends to become a plane and allows more free space, this promotes the convective transfer to be predominant as the average equivalent conductivity shows.

\section{Conclusion}

The phenomenon of natural convection in a porous elliptical annulus that is saturated of Newtonian fluid was studied using a numerical method which uses the 
H. Boulechfar et al.: Mechanics \& Industry 16, 403 (2015)
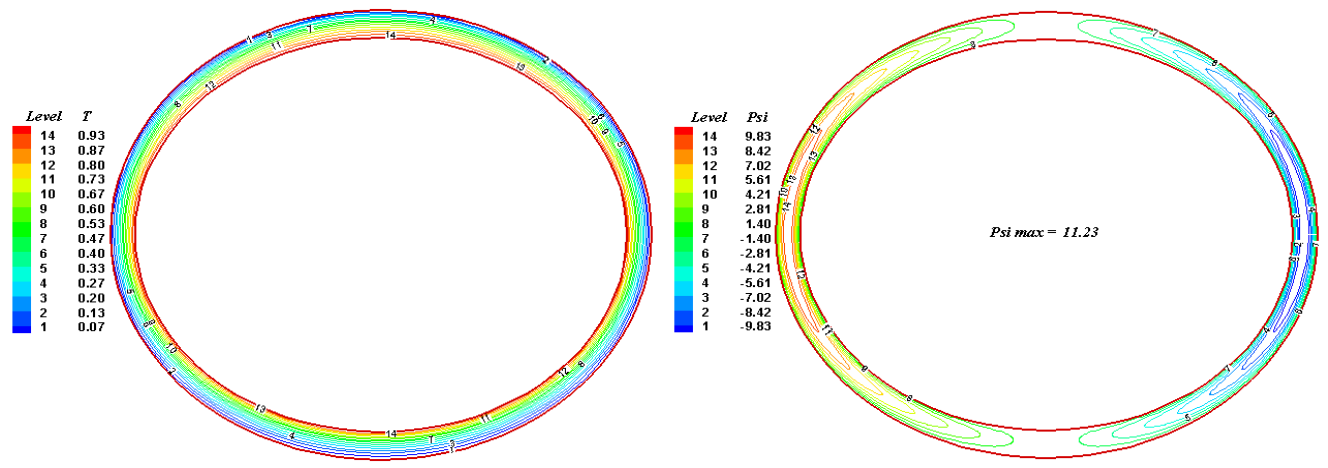

Fig. 3. Isotherms and streamlines for $R a_{m}=500, \alpha=0^{\circ}, e_{1}=0.55$ and $e_{2}=0.5$.
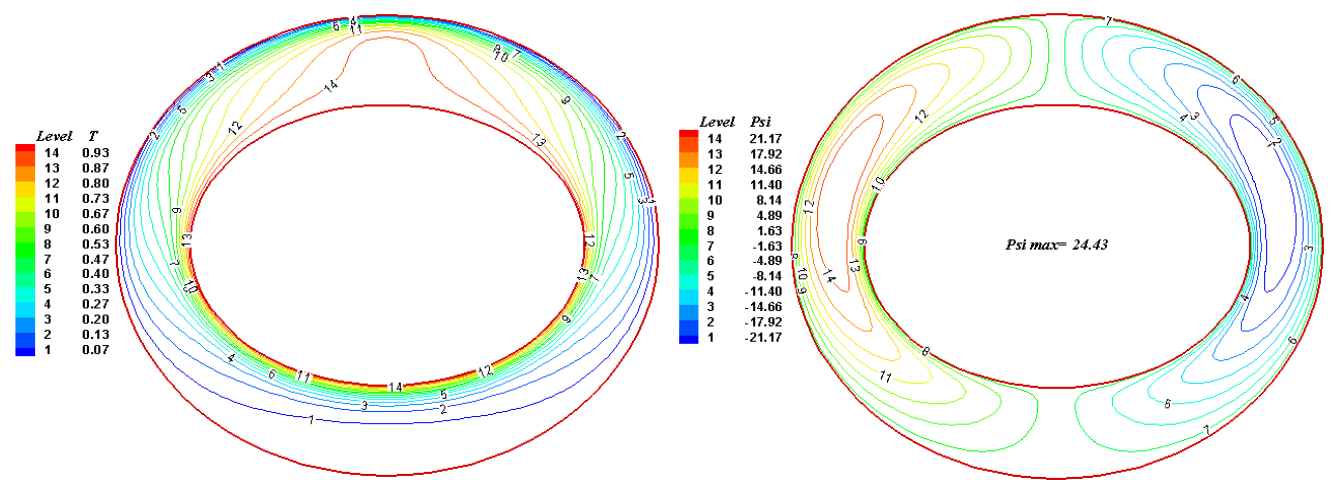

Fig. 4. Isotherms and Streamlines for $R a_{m}=500, \alpha=0^{\circ}, e_{1}=0.688$ and $e_{2}=0.5$.
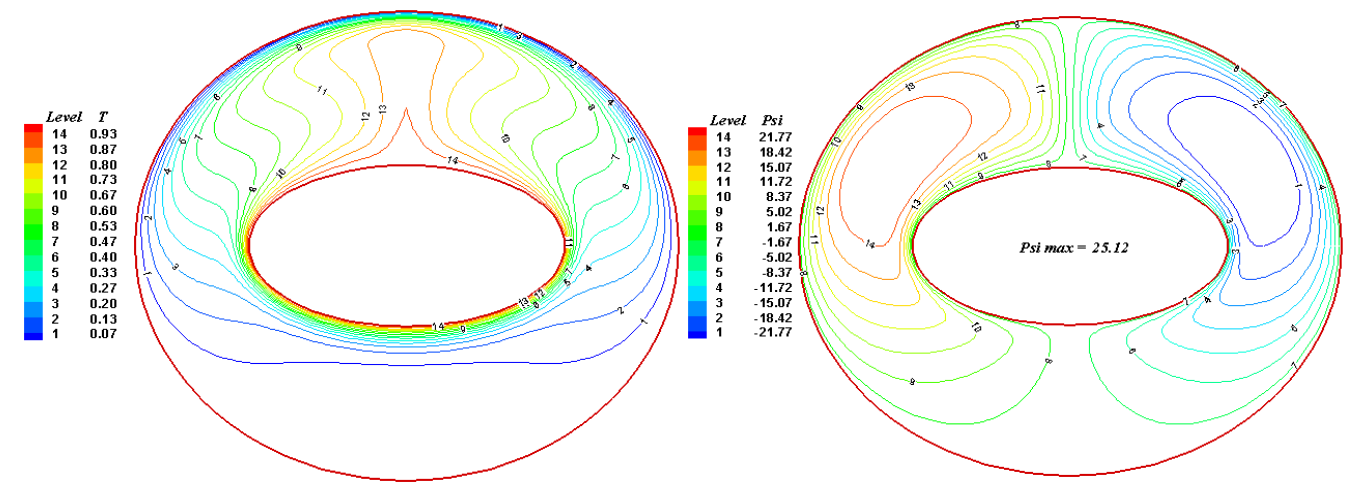

Fig. 5. Isotherms and Streamlines for $R a_{m}=500, \alpha=0^{\circ}, e_{1}=0.86$ and $e_{2}=0.5$.
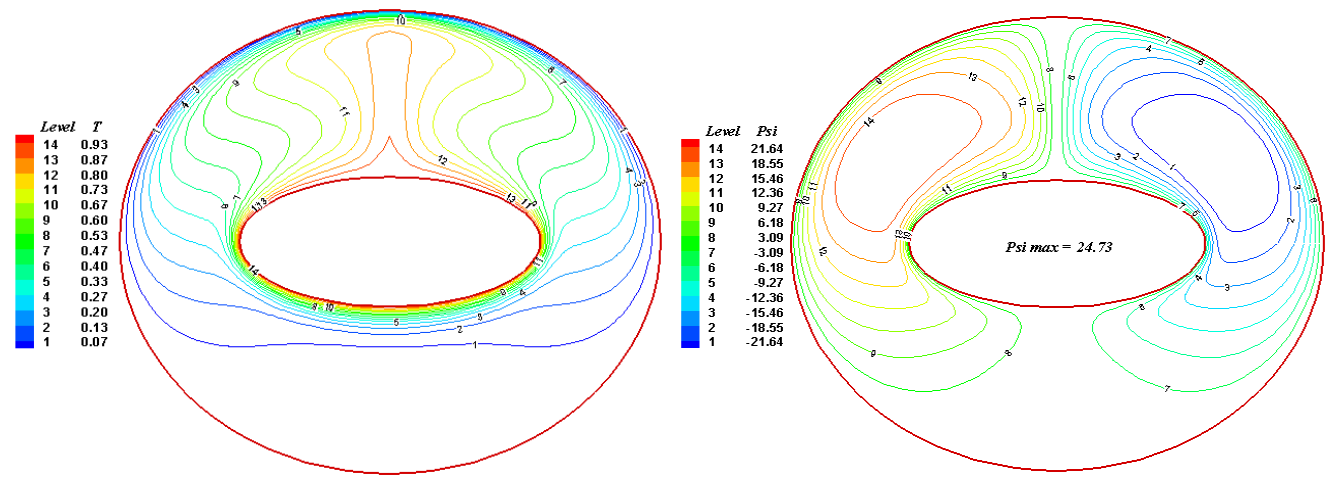

Fig. 6. Isotherms and Streamlines for $R a_{m}=500, \alpha=0^{\circ}, e_{1}=0.9$ and $e_{2}=0.5$. 

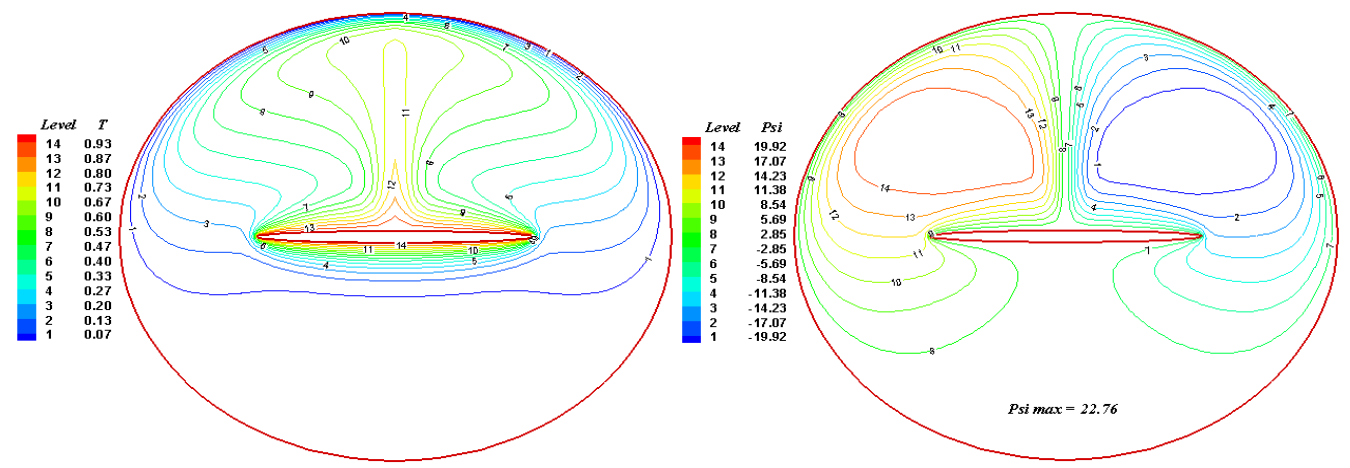

Fig. 7. Isotherms and Streamlines for $R a_{m}=500, \alpha=0^{\circ}, e_{1}=0.999$ and $e_{2}=0.5$.

method of finite volumes, the vorticity-streamline formulation makes it possible to find a good agreement with the literature bidimensional thermal natural convection, in laminar flow and permanent, in an annular space filled with fluid-saturated porous media located between two confocal elliptic cylinders.

We examined, in particular, the influence of the internal eccentricity on the convective mode; the results underline the influence of the geometry on heat transfer that is greatly affected by the eccentricity of the internal cylinder qualitatively and quantitatively. The transfer is better when we considered the most valuable free space between the two cylinders by increasing the eccentricity of the inner elliptical cylinder which promotes the convective mode of heat transfer.

\section{References}

[1] J.R. Philip, Free convection at small Rayleigh number in porous cavities of rectangular, elliptical, triangular and other cross-sections, Int. J. Heat Mass Transfer 25 (1982) 1503-1509

[2] M.M. Elshamy, M.N. Ozisik, Correlation for laminar natural convection between confocal horizontal elliptical cylinders, Numerical Heat Transfer A 18 (1990) 95-112

[3] M.C. Charrier-Mojtabi, Numerical simulation of two- and three-dimensional free convection flows in a horizontal porous annulus using a pressure and temperature formulation, Int. J. Heat Mass Transfer 40 (1997) 1521-1533
[4] J.P.B. Mota, I.A.A.C. Esteves, C.A.M. Portugal, Natural convection heat transfer in horizontal eccentric elliptic annuli containing saturated porous media, Int. J. Heat Mass Transfer 43 (2000) 4367-4379

[5] M. Djezzar, A. Chaker, M. Daguenet, Numerical study of bidimensional steady natural convection in a space annulus between two elliptic confocal ducts, influence of the eccentricity, Rev. Energy. Ren. 8 (2005) 63-72

[6] M. Djezzar, M. Daguenet, Natural steady convection in a space annulus between two elliptic confocal ducts. Influence of the slope angle, J. Appl. Mech. Trans. ASME 72 (2006) 88-95

[7] J.C. Leong, F.C. Lai, Natural convection in a concentric annulus with a porous sleeve, Int. J. Heat Mass Transfer 49 (2006) 3016-3027

[8] K. Khanafer, A. Al-Amiri, I. Pop, Numerical analysis of natural convection heat transfer in a horizontal annulus partially filled with a fluid-saturated porous substrate, Int. J. Heat Mass Transfer 51 (2008) 1613-1627

[9] M. Kumari, G. Nath, Unsteady natural convection from a horizontal annulus filled with a porous medium, Int. J. Heat Mass Transfer 51 (2008) 5001-5007

[10] M. Sankar, Y. Park, J.M. Lopez, Y. Do, Numerical study of natural convection in a vertical porous annulus with discrete heating, Int. J. Heat Mass Transfer 54 (2011) 1493-1505

[11] S.V. Patankar, Numerical heat transfer and fluid flow, Hemisphere, Washington D.C., 1980 